\title{
RED DE TRANSFERENCIA DE CONOCIMIENTO EN LA TRANSFORMACIÓN DIGITAL DEL SECTOR OLIVARERO ANDALUZ SEgÚN LA PERSPECTIVA DE LOS AGENTES DE GENERACIÓN DE CONOCIMIENTO
}

\author{
$\underline{\text { Liliana Reina }}^{\mathrm{a}}$, Carlos Parra ${ }^{\mathrm{b}^{*}}$, Carmen Carmona ${ }^{\mathrm{b}}$, Samir Sayadi ${ }^{\mathrm{b}}$ \\ ${ }^{a}$ Universidad de Córdoba; Dpto. Economía, Sociología y Política Agraria (Córdoba, z52reusm@uco.es). \\ ${ }^{b}$ Instituto de Investigación y Formación Agraria y Pesquera (IFAPA); Area de Economía de la Cadena \\ Alimentaria; Centro Camino de Purchil (Granada, carlos.parra@juntadeandalucia.es, \\ mariac.carmona.torres@juntadeandalucia.es,samir.sayadi@juntadeandalucia.es).
}

\section{Resumen}

La presente comunicación analiza la configuración de la red social de transferencia de conocimiento en el sistema de innovación tecnológica (SIT) asociado a la transformación digital (TD) del sector olivarero de Andalucía. Así, mediante el uso de técnicas de Análisis de Redes Sociales (ARS), y desde la perspectiva de los agentes de generación de conocimiento (Universidades, OPIs), se identifican los principales agentes emisores y receptores de conocimiento en dicho proceso de TD. Los resultados indican que las universidades y los OPIs y los agentes de transferencia de conocimiento figuran como los principales nodos emisores de conocimiento, y destaca su papel como cohesionadores de la red social. Los principales nodos receptores de conocimiento son los olivareros y las cooperativas. La configuración de la red social presenta una densidad alta que implica una relación positiva con la generación de capital social. No obstante, la mayoría de las interacciones establecidas entre los diferentes agentes del sistema de innovación son de intensidad muy débil, lo que afecta negativamente a la cohesión del SIT de la TD del sector olivarero de Andalucía.

Palabras clave: Sistema de innovación tecnológica, digitalización, agentes de innovación, ARS, olivar.

\section{Introducción}

El futuro de las áreas rurales, en diferentes partes de la geografía mundial, se enfrenta a enormes paradojas e incertidumbres, ante las cuales se están originando múltiples respuestas y enfoques desde los actores locales de los territorios para abordarlas, así como desde las políticas públicas. Una de las aproximaciones teóricas recientes a esta problemática, originada en la Unión Europea, está basada en el concepto de territorios inteligentes. Un territorio inteligente se define como un espacio geográfico, rural o urbano, cuyo objetivo es resolver problemas públicos mediante soluciones de base tecnológica en el marco de una asociación entre múltiples agentes de diferentes sectores económicos (Navío-Marco et al., 2020). No obstante, el concepto de territorio desde la concepción de Navío-Marco et al. (2020) podría transcender la idea reduccionista y estática de un mero espacio físico dado y retomar los enfoques de la perspectiva territorial del desarrollo rural, adoptada en la política rural de la Unión Europea (Medeiros and Rauhut, 2020; Sánchez-Zamora et al., 2014). Desde esta perspectiva, el territorio pasa a ser concebido como un proceso de construcción social determinado por los actores locales y sus interacciones para valorizar los recursos territoriales (Sánchez-Zamora et al., 2014). Las formas de interacción social, convergentes y legitimadas, inciden en los procesos de organización territorial (D'Aquino, 2002) y en la configuración del capital social. De esta manera, podemos construir un nuevo concepto basado en las dos construcciones teóricas anteriores, que se podría denominar 'territorios rurales digitales' (TRD), que se pueden definir, basados en el enfoque territorial, como espacios rurales en que los actores locales hacen uso de innovaciones digitales para mejorar la eficiencia de sus procesos sociales y económicos. El concepto de TRD podría facilitar el estudio de los procesos de innovación en el marco de la transformación digital (TD) en las áreas rurales. En este contexto, el principal objetivo de este estudio es comprender cómo las redes de actores y el potencial capital social que se configura, facilitan u obstaculizan la formación de un sistema de innovación tecnológica (SIT). Más concretamente, a través del Análisis de Redes Sociales (ARS) analizamos el papel de los agentes vinculados al SIT de la TD en el sector olivarero en Andalucía. Dada la amplitud del concepto de SIT, nos centramos en la función de transferencia (emisión y/o recepción) de conocimiento.

\section{Metodología}

\subsection{Análisis de Redes Sociales (ARS)}

Esta investigación hace uso de la metodología de ARS, también denominada Análisis Estructural, que consiste en un conjunto de técnicas de análisis de las relaciones entre actores y las estructuras sociales que 
surgen de estas. Una red está conformada por dos elementos básicos: los actores (nodos/puntos en la red) y las relaciones que se establecen entre ellos (aristas/líneas en la red). El ARS hace uso del lenguaje matemático de la teoría de grafos, de matrices y de álgebra relacional (Sanz Menéndez, 2003; Semitiel García and Noguera Méndez, 2004). El ARS permite calcular una serie de indicadores que permiten caracterizar la estructura de una red y la relevancia de los nodos. Los indicadores de estructura (cohesión) son medidas de la red completa, frente a las medidas de los nodos que son indicadores individuales (Tabla 1). Así, se pueden identificar redes cerradas en la que todos los nodos están conectados (Buciega and Esparcia, 2013; Coleman, 2003), o redes con puente, en las que las relaciones no son tan fuertes, pero existen nodos conectores (Buciega and Esparcia, 2013).

Tabla 1. Principales indicadores ARS

\begin{tabular}{|c|c|c|c|}
\hline \multicolumn{2}{|r|}{ Estructura de la red } & \multicolumn{2}{|r|}{ Posición de los nodos } \\
\hline Grado medio & $\begin{array}{l}\text { Media aritmética de las relaciones que tiene cada } \\
\text { nodo }\end{array}$ & Grado & $\begin{array}{l}\mathrm{N}^{o} \text { de conexiones de un nodo. Puede ser de } \\
\text { entrada o salida }\end{array}$ \\
\hline Diámetro de la red & Distancia más grande entre dos nodos & Cercanía & El nodo que está más cerca de todos los nodos \\
\hline Densidad del grafo & $\begin{array}{l}\mathrm{N} .^{\circ} \text { de conexiones establecidas en la red sobre el } \\
\text { total de conexiones posibles. La densidad mide } \\
\text { cómo de cerca está una red de ser completa. Una } \\
\text { red completa tiene todas las aristas (relaciones) } \\
\text { posibles y una densidad igual a } 1\end{array}$ & Intermediación & $\begin{array}{l}\text { Las veces que un nodo está en la ruta que } \\
\text { conecta a todos los demás nodos }\end{array}$ \\
\hline
\end{tabular}

Elaboración propia a partir de Scott (2017)

\subsection{Obtención de la información}

Se ha obtenido información primaria en base a entrevistas a 14 personas expertas y/o vinculadas a la TD del sector olivarero de Andalucía, pertenecientes a universidades o centros de investigación (agentes de generación de conocimiento), entre agosto y octubre de 2020. Se utilizó un cuestionario estructurado en el que se pedía identificar las interacciones de 21 agentes previamente definidos en el SIT de la TD del sector olivarero de Andalucía (Tabla 2). En cada interacción calificaron la intensidad de las relaciones entre agentes en una escala entre 0 y $9(0=$ ninguna relación, $9=$ muy alta intensidad de relación $)$.

Tabla 2. Agentes del SIT de la TD del sector olivarero de Andalucía

\begin{tabular}{|l|l|}
\hline 1. Olivarero & 12. Interprofesional del aceite de oliva \\
2. Cooperativa / grupo cooperativo & 13. Consultor privado \\
3. Grupo no cooperativo (Interóleo, etc.) & 14. Empresa suministradora de inputs agrarios \\
4. Denominación de Origen Protegida (DOP) & 15. Empresa de tecnologías digitales \\
5. Asociación agraria (SAT, ATRIA, API) & 16. Agente de generación de conocimiento (Universidad, OPI, etc.) \\
6. Organización agraria (UPA, COAG, ASAJA) & 17. Agente de transferencia del conocimiento (Centro tecnológico, etc.) \\
7. Refinería & 18. Agente de gestión del conocimiento (IDEA, RETA, etc.) \\
8. Envasadora & 19. Administración pública (Delegación agraria, OCA, Diputación, etc.) \\
9. Agente de distribución & 20. Institución financiera (banco, caja de ahorros) \\
10. Grupo de Desarrollo Rural (GDR) & 21. Medios de comunicación científicos y divulgativos (revistas, internet, etc.) \\
11. Patrimonio Comunal Olivarero & \\
\hline
\end{tabular}

\subsection{Análisis de la información}

Para procesar la información primaria y realizar el ARS se ha utilizado el software de código abierto y gratuito Gephi 0.9.2 (https://gephi.org/).

\section{Resultados}

\subsection{Características de la red}

La red resultante del SIT de la TD en el sector olivarero en Andalucía está compuesta por los 21 agentes identificados previamente y 439 conexiones establecidas entre ellos. Un primer análisis se centra en la red 'global' que se basa en los vínculos acumulados, independientemente de su calidad (es decir, tipo e 
intensidad de las conexiones) (Figuras 1 y 2). La densidad de la red es 1, lo que indica que todos los agentes están conectados entre sí. Además, se trata de una red completa ya que no existe ningún actor desconectado. En cuanto a la centralización, destaca que no hay un único agente central, sino que existe un conjunto de agentes en el centro de la red, que configuran una red descentralizada, lo que indica que la toma de decisiones está sustancialmente distribuida a través del SIT.

\subsection{Agentes en la transferencia de conocimiento}

La Figura 1 muestra que los principales agentes emisores de conocimiento a otros agentes son, de mayor a menor grado: 16. Agente de generación de conocimiento, 17. Agente de transferencia del conocimiento, 21. Medios de comunicación científicos y divulgativos, 19. Administración pública y 2. Cooperativa / grupo cooperativo. En la Figura 2 se puede comprobar que los principales agentes receptores de conocimiento son, de mayor a menor grado: 1 . Olivarero, 2. Cooperativa / grupo cooperativo, 3 . Grupo no cooperativo, 4. Denominación de Origen Protegida, y 17. Agente de transferencia del conocimiento. Es importante destacar el doble papel que tienen los agentes 2. Cooperativa / grupo cooperativo y 17. Agente de transferencia del conocimiento, tanto como emisores como receptores de conocimiento en la TD.

Gráfico 1. Red social del SIT de la TD en el sector olivarero de Andalucía-Agentes emisores de conocimiento

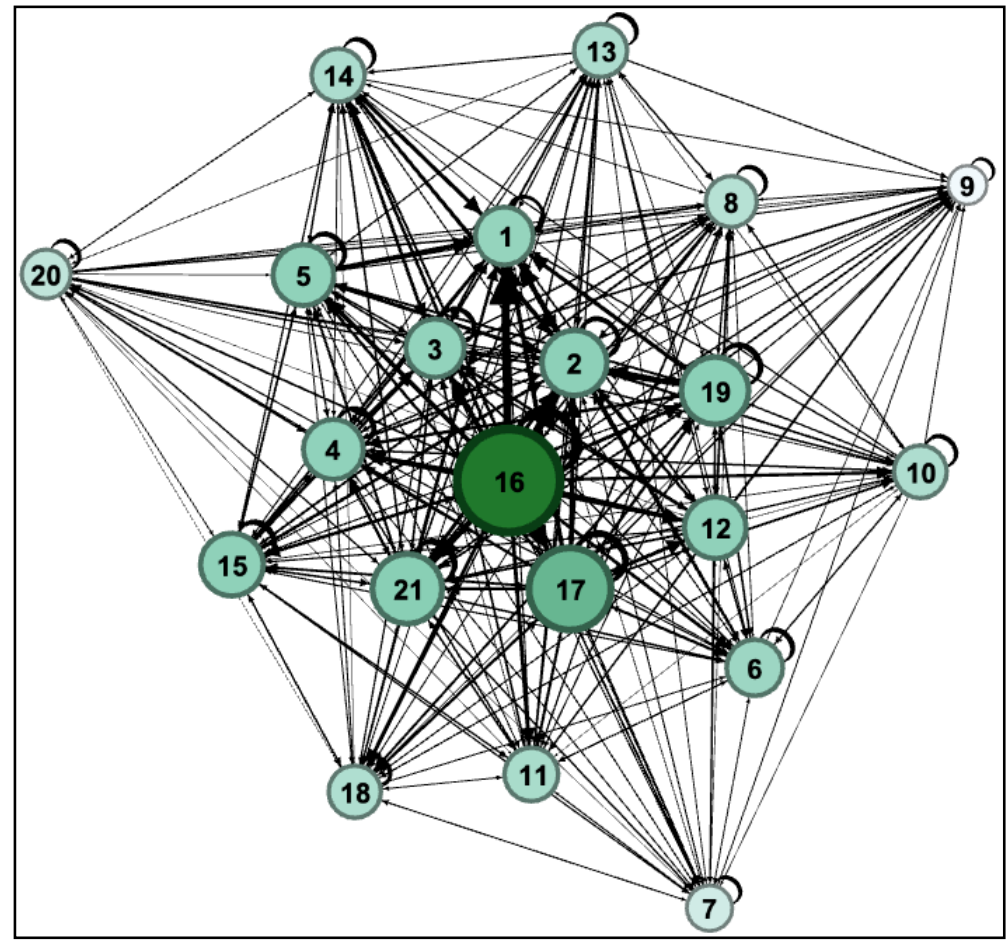

Nota: El tamaño y color de los nodos indica el grado de emisión (salida) de información, cuanto más grande y oscuro sea el nodo, mayor será su grado de transmisión de información Se ha utilizado la distribución ForceAtlas con fuerza de repulsión 200000 
Gráfico 2. Red social del SIT de la TD en el sector olivarero de Andalucía-Agentes receptores de conocimiento

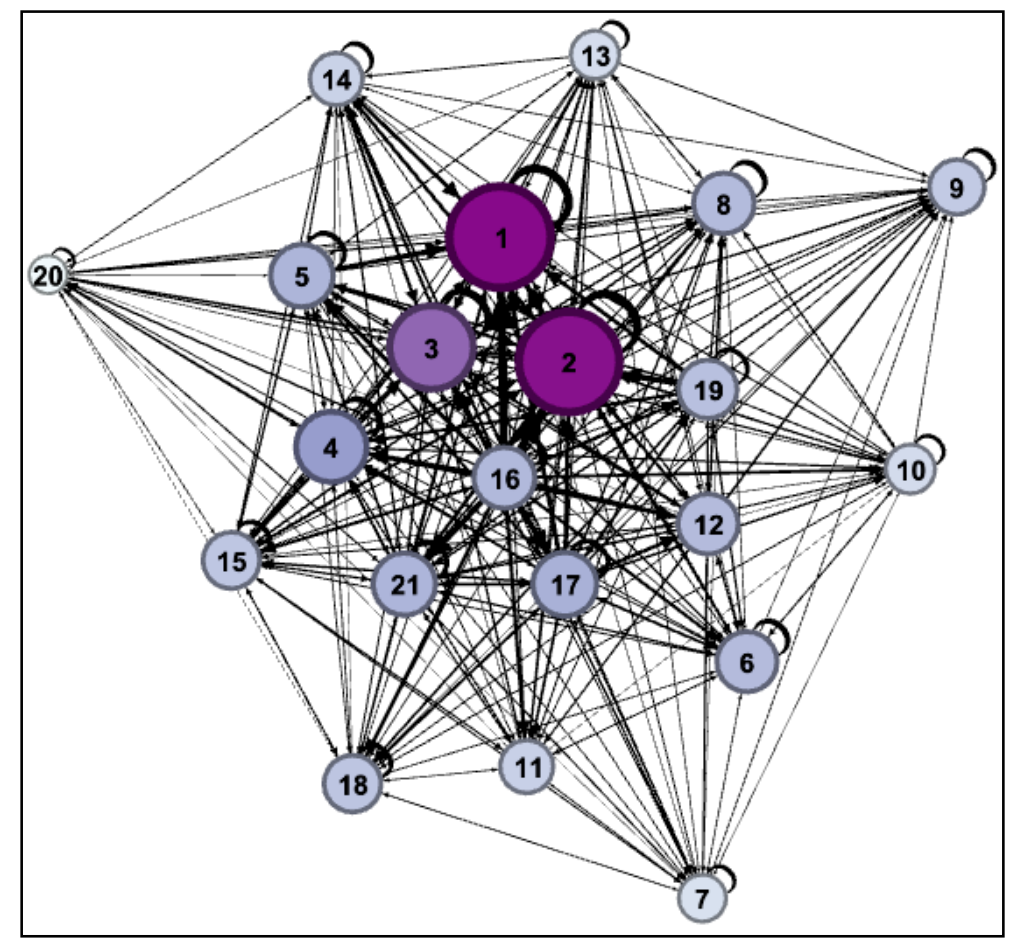

Nota: El tamaño y color de los nodos indica el grado de recepción (entrada) de información, cuanto más grande y oscuro sea el nodo, mayor será su grado de transmisión de información. Se ha utilizado la distribución ForceAtlas con fuerza de repulsión 200000

\subsection{Conexiones en la transferencia de conocimiento}

Las conexiones entre los agentes de la red se han clasificado en diferentes categorías según su intensidad. Destaca que el 87,98 \% de las conexiones se clasifican como muy débiles (rango de peso de la arista: 0 $1,80), 10,66 \%$ como débiles $(1,81-3,60)$, y sólo el $0,45 \%$ como fuertes $(5,41-7,20)$, y no existe ninguna conexión en la categoría de peso medio $(3,61-5,40)$ o muy fuerte $(7,21-9,00)$.

\section{Conclusiones}

El ARS ha permitido caracterizar la red social de transferencia de conocimiento en el SIT asociado a la TD del sector olivarero de Andalucía, desde la perspectiva de los agentes de generación de conocimiento. Los resultados indican que entre los agentes que destacan por su papel como emisores de conocimiento se encuentran los agentes de generación de conocimiento, los agentes de transferencia del conocimiento y los medios de comunicación científicos y divulgativos. Por otro lado, los principales agentes receptores de conocimiento son los olivareros, las cooperativas o grupos cooperativos, y los grupos no cooperativos. Es importante destacar el doble papel que tienen las cooperativas o grupos cooperativos, y los agentes de transferencia de conocimiento, tanto como emisores como receptores de conocimiento. La densidad de las relaciones, medida por el número de relaciones que se establecen en la red en el SIT de la TD, es bastante alta, por lo que tiene un alto potencial para la creación de capital social. Además, se trata de una red completa, sin ningún actor desconectado, y descentralizada, lo que indica que la toma de decisiones está distribuida a través de la red. Según esta configuración de la red del SIT de la TD del sector olivarero en Andalucía, se puede considerar que tiene un alto potencial para la creación de capital social. No obstante, pese a la alta densidad de la red la intensidad de las relaciones establecidas es, en general, muy débil. Así, un incremento de las interacciones, en número e intensidad, entre los agentes de la red del SIT, favorece la formación de capital social, y con ello podría aumentar la eficiencia de los procesos de transferencia de conocimiento en la TD, y facilitar acciones coordinadas que potencien la acción colectiva y la cooperación para beneficio mutuo de los actores. Finalmente, futuras investigaciones en esta materia podrían profundizar en los indicadores de caracterización de la red del SIT, ajustando las medidas al tipo de SIT, y complementar este estudio, en el que se ha analizado la dimensión interna (cómo se relacionan los agentes 
del sistema) del SIT de la TD en el sector olivarero de Andalucía, con un análisis exhaustivo de la capacidad del SIT para generar capital social a partir de sus relaciones internas y externas.

Bibliografía

Buciega, A., Esparcia, J., 2013. "Desarrollo, Territorio y Capital Social. Un análisis a partir de dinámicas relacionales en el desarrollo rural". Redes. Rev. Hisp. para el análisis redes Soc. 24, 81-113.

Coleman, J., 2003. "Social Capital in the Creation of Human Capital". En: Cross, R., Parker, A., Lisa, S. (Eds.): Networks in the Knowledge Economy. Oxford, pp. 57-81.

D'Aquino, P., 2002. "Le territoire entre espace et pouvoir: Pour une planification territoriale ascendente". Espac. Geogr. 31, 3-22. https://doi.org/10.3917/eg.311.0003

Medeiros, E., Rauhut, D., 2020. "Territorial Cohesion Cities: a policy recipe for achieving Territorial Cohesion?" Reg. Stud. https://doi.org/10.1080/00343404.2018.1548764

Navío-Marco, J., Rodrigo-Moya, B., Gerli, P., 2020. "The rising importance of the "Smart territory" concept: definition and implications". Land use policy 99.

Sánchez-Zamora, P., Gallardo-Cobos, R., Ceña-Delgado, F., 2014. "Rural areas face the economic crisis: Analyzing the determinants of successful territorial dynamics". J. Rural Stud. 35, 11-25.

Sanz Menéndez, L., 2003. "Análisis de redes sociales: o cómo representar las estructuras sociales subyacentes". Apunt. Cienc. y Tecnol. 7, 10.

Scott, J., 2017. Social network analysis, 4th ed. SAGE publication Ltd, London.

Semitiel García, M., Noguera Méndez, P., 2004. "Los Sistemas Productivos Regionales desde la perspectiva del Análisis de Redes". REDES- Rev. Hisp. para el análisis redes Soc. 6, 1-26.

\section{Agradecimientos}

Los autores desean expresar su agradecimiento por el apoyo financiero recibido del Instituto de Investigación y Formación Agraria y Pesquera (IFAPA), a través del proyecto de investigación 'Transformación digital del sector olivarero en Andalucía: Análisis sistémico, estructural y funcional para favorecer su desarrollo (digitalOli)' (PR.AVA.AVA2019.009), cofinanciado en un 80\% por el Fondo Europeo de Desarrollo Regional dentro del Programa Operativo FEDER de Andalucía 2014-2020. 